\title{
Proteomic Analysis Revealed Underlying Biological Pathways Associated with Hormetic Response of Hormone- Positive Breast Cancer Cell Line Exposed to Low-Dose Flavonoid Mixture
}

\author{
Mete Bora Tuzuner ${ }^{1}$ (D, Ayse Begum Ceviz² (D) \\ 'Acıbadem Labmed Medical Laboratories, Research and Development Center, Istanbul, Turkey \\ 2Istanbul Health and Technology University, Faculty of Medicine, Department of Medical Genetics, Istanbul, Turkey
}

ORCID IDs of the authors: M.B.T. 0000-0001-8924-4850; A.B.C. 0000-0002-3635-8421

Please cite this article as: Tuzuner MB, Ceviz AB. Proteomic Analysis Revealed Underlying Biological Pathways Associated with Hormetic Response of Hormone-Positive Breast Cancer Cell Line Exposed to Low-Dose Flavonoid Mixture. Eur J Biol 2021; 80(2): 145-153. DOI: 10.26650/EurJBiol.2021.1017807

\section{ABSTRACT}

Objective: A considerable level of evidence has accumulated about the breast cancer risk-reducing effect of consuming specific flavonoids, through the increasing amount of research and epidemiologic studies. Different flavonoids may have different cellular bioavailability and favor, i.e., the occurrence of a hormetic effect, thus it is important to evaluate breast cancer cells' response to different doses of flavonoids. This study aims to investigate the alterations of the biological pathways in a hormone-positive ( $\mathrm{HR}+$ ) breast cancer cell line as a resemblance for the most common breast cancer subtype, related to the low-dose exposure of the flavonoids.

Materials and Methods: Different levels of doses were applied to MCF-7 breast cancer cells. In order to determine cellular proliferation, WST-1 analysis was conducted. The highest proliferation was observed with cell lines exposed to a low-dose flavonoid mixture and these were selected for further analysis. Intracellular protein expression were investigated by peptide analysis on a nano LC-MS/MS platform. A protein-protein interaction network and pathway analysis were conducted for the proteins expressed differently between the groups.

Results: A total of 214 proteins were identified and 36 proteins with significant alterations ( $\geq 1.2$-fold change, $p \leq 0.05)$ were detected. Significant changes were observed in the pathways related to carbon metabolism, amino acid biosynthesis, splicing mechanism, mitochondrial protein import and translation elongation pathways.

Conclusion: Our study demonstrated that flavonoids can have a hormetic effect which can initially alter metabolic pathways vital for cell proliferation and survival. These pathways may include potential targets for enhancing the anticancer activity of the flavonoids.

Keywords: Flavonoids, breast cancer, hormesis, proteomics

\section{INTRODUCTION}

Breast cancer has the highest incidence rate and occupies second place after lung cancer for the mortality rates in women worldwide (1). One of the best ways to reduce the global burden of breast cancer is prevention. Maintaining a healthy diet which is rich in flavonoids has been suggested to achieve that goal (2). Flavonoids are polyphenolic compounds which are highly represented in many plants, fruits, vegetables and propolis. Especially, propolis has a very important place as a medicine in Eastern Europe and Asian regions (3). Because of the wide range of pharmacological properties, it is commonly used by people as a dietary supplement whether being in good health or suffering from a disease like cancer (4). 
Studies have shown a significant difference between the breast cancer incidence rates of Asian and Western populations (5). Thus, the differences of dietary habits, regarding the flavonoid consumption tendencies among populations can be suggested as a factor for this phenomenon. Furthermore, the breast cancer prevalence increase, among Asian women who migrate to western countries, may also be considered as evidence for the protective effect of flavonoids against breast cancer (6).

Despite the anticancer effects of flavonoids, their bioavailability is generally low and can vary dramatically among different flavonoid classes as a consequence of Phase 2 metabolism. Most flavonoids go through several steps of ingestion such as glucuronidation, methylation and sulfation in the small intestine and liver before their conjugated metabolites can be found in plasma (7). Overall, flavonoids' metabolites have lower bioactivity than the parent molecules. Having a high molecular weight and a complex molecular structure are also factors for the attenuated bioavailability levels (8). Therefore, in vivo bioavailability of flavonoids must be taken into consideration while assessing their anticancer bioactivity via in vitro systems.

Several studies have reported the anticancer action of different flavonoids with cell culture and animal models $(9,10)$. However, when the dose-response relationship is considered, an increase of tumor cell proliferation was observed at low-dose flavonoid exposures (11). This biphasic effect, so called "hormesis", of some flavonoids such as daidzein, genistein, kaempferol, quercetin, luteolin and resveratrol, have been demonstrated in several studies $(12,13)$. Up to date literature points out that exposure to such flavonoids may cause cells to synthesize protective proteins against upcoming stress, including increased energy demand, free radical production and ion fluxes via triggering adaptive response pathways (12). Furthermore, studies have suggested that the transactivation of the estrogen receptor may be one of the reasons for the hormetic effect (13). However, there is still insufficient data presented to elucidate the responsible biphasic response mechanisms of the breast cancer cells exposed to multiple flavonoids.

In the current study, we investigated the hormetic effect of a selected mixture of flavonoids (henceforth referred to as "Flavonoid Mix") which we have the patent for pending formulation regarding our research group's previous studies $(9,14)$. By investigating the proliferation part of the hormetic effect on the MCF-7 cell line, we aimed to provide insights about hormone driven breast cancer cells' response mechanisms for the non-cytotoxic dose of the Flavonoid Mix (FM). Our findings will provide important understanding on how the commonest molecular subtype of breast cancer rewires its metabolic pathways in response to the initial stress caused by flavonoid exposure that may not be obtained from high-dose studies alone.

\section{MATERIALS AND METHODS}

\section{Materials}

Acetonitrile (LC-MS grade) and water (LC-MS grade) were purchased from Merck (EMD Millipore). Dithiothreitol (DTT) and io- doacetamide (IAA) were purchased from Sigma-Aldrich. Formic acid (FA) was obtained from Fluka. Sequencing grade modified trypsin (proteomic grade) was acquired from Thermo Scientific. The MCF-7 cell line was obtained from American Type Cell Collection (MD, USA). Dulbecco's Modified Eagle's Medium (DMEM) was purchased from Sigma Aldrich. Trypsin-EDTA (0.05\%), penicillin/streptomycin and FBS were obtained from Biochrome.

\section{Culture of MCF-7 Cells}

The ER/PR (+), HER2 (-) human breast cancer cell line, MCF-7, was purchased from American Type Culture Collection (ATCC, Rockville, MD) and the rest of the cell culture related materials were obtained from Biochrome (Berlin, Germany). Cells were maintained in high glucose DMEM and all were supplemented with $10 \%$ fetal bovine serum (FBS), $1 \%$ glutamine, and $1 \%$ penicillin/streptomycin. They were incubated at $37^{\circ} \mathrm{C}$ in a $5 \% \mathrm{CO}_{2}$ humidified atmosphere. When the density of the cells reached $70-80 \%$, subculturing was done and sixth-eighth passages were used in the experiment.

\section{Preparation of the Flavonoid Mix}

The "Flavonoid Mix" (FM) was originated from our research group's previous studies on propolis extracts $(9,14)$. Seven flavonoids, including apigenin and luteolin (>95\% HPLC, Sigma-Aldrich, Germany) were used in the preparation of the FM (patent pending). Master stock solutions were created by dissolving object in $60 \%$ ethanol with a sonicator. Then, the doses to be administered in the experiment were prepared by diluting object in a medium containing $3 \%$ FBS. FBS of $3 \%$ was preferred since high percentages of FBS such as 10\%, although appropriate for rapid cell growth, deflects the WST-1 reading results (15). Before the solutions were administered, they were made ready for use by passing them through $0.22 \mu \mathrm{m}$ filters. The FM was formed by combining the compounds of which the $\mathrm{IC}_{50}$ values were determined by applying object to the cells one by one, each containing the $\mathrm{IC}_{50}$ concentration, and this mixture contained $117 \mu \mathrm{g} / \mathrm{ml}$ flavonoids in total. Other doses (16, 26, 63, 90 $\mu \mathrm{g} / \mathrm{ml}$ ) were then prepared with dilutions.

\section{Cell Proliferation Analysis}

The cell proliferation reagent WST-1 (Roche, Manheim, Germany) was used for the cell proliferation analysis. Vi-Cell XR Cell Viability Analyzer (Beckman Coulter, Brea, CA, USA) was employed for the cell counting process. $1 \times 10^{4}$ cells were seeded per well with medium supplemented with 3\% FBS in 96 well plates (Greiner Bio-one, Kremsmünster, Austria). Fresh medium replacement was done after overnight adherence of the cells. Then, doses $(16,26,63,90,116 \mu \mathrm{g} / \mathrm{mL})$ of FM were applied to the cells to determine the effects at different time intervals $\left(24^{\text {th }}, 48^{\text {th }}\right.$, and $\left.72^{\text {nd }} \mathrm{h}\right)$. WST- 1 reagent $(10 \mu \mathrm{L})$ was added to each well at the designated time intervals and afterwards 2 hours of incubation was carried out at $37^{\circ} \mathrm{C}$ in $5 \% \mathrm{CO}_{2}$. Multiscan ELISA reader (Thermo Fisher Scientific, Waltham, MA, USA) was used to measure the absorbance of the wells at $450 \mathrm{~nm}$ with the reference wavelength set at $620 \mathrm{~nm}$ in order to detect the formazan formation. Control cells' viability were accepted as $100 \%$. Data are expressed as percentages of 
absorbance readings compared to control wells on a relative proliferation index scale (Mean \pm standard deviation). All tests were performed in triplicate.

\section{LC-MSMS Analysis}

Tryptic peptides were generated according to the Filter Aided Sample Preparation Protocol (FASP) (16). Cells were scraped from cell culture plates and washed twice with cold $50 \mathrm{mM}$ ammonium bicarbonate and lysed in a $50 \mathrm{mM}$ ammonium bicarbonate solution via ultrasonic homogenization ( $5 \mathrm{~s}$ on, $5 \mathrm{~s}$ off, 3 cycles). The mixture was centrifuged at 15,000 rpm and the protein concentration in the supernatant was measured based on NanoDrop. Thirty $\mu \mathrm{L}$ of protein mixture containing $100 \mu \mathrm{g}$ protein was transferred to a $30 \mathrm{kDa}$ cut-off spin filter and mixed with $200 \mu \mathrm{L}$ of $6 \mathrm{M}$ urea and centrifuged at $14,000 \times \mathrm{g}$ for 15 min. The process was repeated twice. The flow through was discarded and the proteins were alkylated with $10 \mathrm{mM}$ iodoacetamide in the dark at room temperature (RT) for $20 \mathrm{~min}$. The protein mixture was washed first with $200 \mu \mathrm{L}$ of $6 \mathrm{M}$ urea and later twice with $100 \mu \mathrm{L}$ of $50 \mathrm{mM} \mathrm{AmBic}$ solution. The mixture was incubated with 1:100 (trypsin:protein) ratio of MS grade trypsin overnight. Peptides were eluted from the spin column first with $50 \mathrm{mM}$ Ambic solution and later with $0.5 \mathrm{M} \mathrm{NaCl}$. The flow through was collected and lyophilized. The concentration of the tryptic peptides was measured with a NanoDrop spectrophotometer and samples were prepared at a concentration of $100 \mathrm{ng} / \mu \mathrm{L}$.

In LC-MS/MS analysis, Symmetry C18 $(5 \mu \mathrm{m}, 180 \mu \mathrm{m}$ i.d. $\times 20$ $\mathrm{mm}$ ) column was used for trapping tryptic peptides. Sixty minutes of $\mathrm{ACN}$ gradient ( $4 \%$ to $40 \% \mathrm{ACN}, 0.3 \mu \mathrm{L} / \mathrm{min}$ flow rate) was applied for elution on a CSH C18 $(1.7 \mu \mathrm{m}, 75 \mu \mathrm{m}$ i.d. $\times 250 \mathrm{~mm})$ analytical nano column. Data collection and processing was performed as it is stated in our previous studies (17).

\section{Bioinformatic Analysis}

To seek potential interactions between identified proteins according to low-dose flavonoid exposure, the STRING database (http://string-db.org/, v11.0) was employed. In order to construct the PPI network, text mining, experiments, database co-expression, neighborhood, gene fusion, and co-occurrence were selected as active interaction sources. Results with a high confidence level (high confidence $=0.70$ ) were taken into consideration. Interpretation and analysis of pathways were performed with REACTOME (http://www.reactome.org, v76.0) and KEGG (Kyoto Encyclopedia of Genes and Genomes, https:// www.genome.jp/kegg/, Release 98.0) databases. Proteins showing significant differences were categorized based on biological processes and molecular function using AmiGO (18).

\section{RESULTS}

\section{Effects of the FM on Cell Viability and Proliferation}

Normal growth features expected under standard in vitro culture conditions were observed among all MCF-7 cells. Cells were grown in increasing concentration of FM, ranging from 16 $\mu \mathrm{g} / \mathrm{mL}$ to $117 \mu \mathrm{g} / \mathrm{mL}$ for up to 3 days and cell viability was determined using the standard WST-1 assay. Our analysis showed that FM had a hormetic dose response on MCF-7 cells. We observed the highest viability relative to the untreated cells at the $24^{\text {th }}$ hour with a dose of $\left.16 \mu \mathrm{g} / \mathrm{ml} \mathrm{FM} \mathrm{(} \mathrm{135 \% ,} \mathrm{Figure} 1\right)$, as well as the highest viability difference ( 97\%) compared to the next dose. Therefore, cells exposed to $16 \mu \mathrm{g} / \mathrm{mL}$ for 24 hours were selected for further proteomic analysis.

\section{Proteome Analysis of the MCF-7 Cells with Maximum Hormetic Response}

In order to analyze the protein expression differences between FM treated MCF-7 cell line and controls, mass spectrometry-based proteomics methods were used. Progenesis QI-P was employed to perform charge state deconvolution and deisotoping and 214 proteins were identified (data not shown) and 36 of them were detected as being significantly altered (fold change $>1.2$ and $p<0.05$ ) in the MCF-7 cell line treated with 16 $\mu \mathrm{g} / \mathrm{mL}$ flavonoid mixture at the $24^{\text {th }}$ hour compared to controls (Table 1). Stress-70 protein (HSPA9) was ranked top amongst the upregulated proteins. On the other hand, Anterior gradient protein 2 homolog (AGR2) was found to be the most downregulated compared to control samples. Overall, the majority of downregulated proteins assume roles in the response to unfolded proteins, RNA binding and catalytic step 2 spliceosome. Ribose phosphate biosynthetic process, cadherin binding and
A

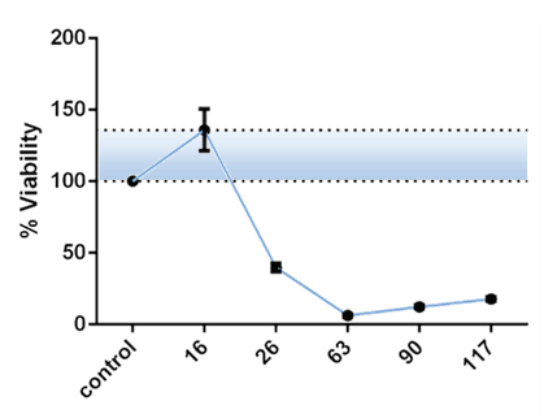

Doses $(\mu \mathrm{g} / \mathrm{ml})$
B

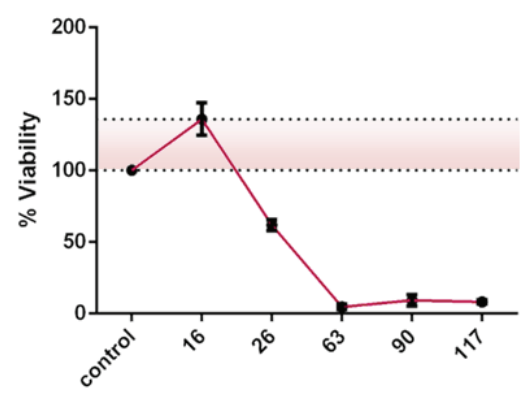

Doses $(\mu \mathrm{g} / \mathrm{ml})$

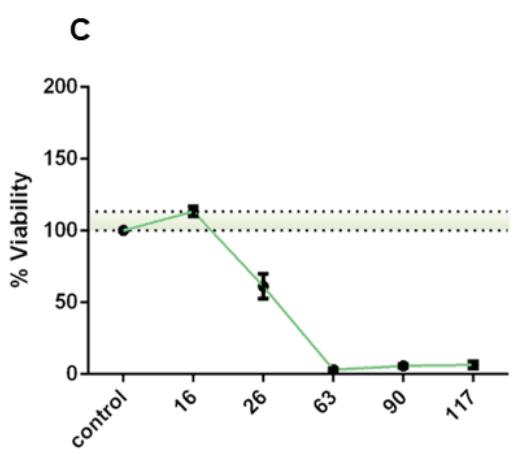

Doses $(\mu \mathrm{g} / \mathrm{ml})$

Figure 1. Effects of the flavonoid mixture on the viability of MCF-7 breast cancer cells. 24 hours (A), 48 hours (B), and 72 hours (C) of exposure are represented. 
Table 1. Significantly altered proteins in MCF-7 cell line exposed to low-dose FM for 24 hours

\begin{tabular}{|c|c|c|c|c|}
\hline Accession no & Gene symbol & Description & Fold Change & $\begin{array}{c}\text { Anova } \\
\text { (p value) }\end{array}$ \\
\hline \multicolumn{5}{|c|}{ UPREGULATED (TREATED vs CONTROL) } \\
\hline P38646 & HSPA9 & Stress-70 protein & 7.87 & 0.0002 \\
\hline P38919 & EIF4A3 & Eukaryotic initiation factor $4 \mathrm{~A}-\mathrm{III}$ & 3.95 & 0.0109 \\
\hline Q5T200 & ZC3H13 & Zinc finger $\mathrm{CCCH}$ domain-containing protein 13 & 3.06 & 0.0139 \\
\hline P34897 & SHMT2 & Serine hydroxymethyltransferase & 2.48 & 0.0079 \\
\hline P62910 & RPL32 & $60 S$ ribosomal protein $\mathrm{L} 32$ & 2.44 & 0.0142 \\
\hline P22626 & HNRNPA2B1 & Heterogeneous nuclear ribonucleoproteins A2/B1 & 1.71 & 0.0315 \\
\hline P02545 & LMNA & Prelamin-A/C & 1.70 & 0.0257 \\
\hline P06733 & ENO1 & Alpha-enolase & 1.65 & 0.0305 \\
\hline P10809 & HSPD1 & 60 kDa heat shock protein & 1.62 & 0.0123 \\
\hline Q13263 & TRIM28 & Transcription intermediary factor 1-beta & 1.61 & 0.0037 \\
\hline P05787 & KRT8 & Keratin type II & 1.58 & 0.0166 \\
\hline P09651 & HNRNPA1 & Heterogeneous nuclear ribonucleoprotein A1 & 1.40 & 0.0206 \\
\hline P04792 & HSPB1 & Heat shock protein beta-1 & 1.39 & 0.0131 \\
\hline P25705 & ATP5A1 & ATP synthase subunit alpha & 1.27 & 0.0425 \\
\hline \multicolumn{5}{|c|}{ DOWNREGULATED (TREATED vs CONTROL) } \\
\hline 095994 & AGR2 & Anterior gradient protein 2 homolog & 12.41 & 0.0004 \\
\hline Q15365 & PCBP1 & Poly(rC)-binding protein 1 & 7.77 & 0.0008 \\
\hline 014745 & SLC9A3R1 & $\mathrm{Na}(+) / \mathrm{H}(+)$ exchange regulatory cofactor NHE-RF1 & 4.67 & 0.0083 \\
\hline P11413 & G6PD & Glucose-6-phosphate 1-dehydrogenase & 4.30 & 0.0009 \\
\hline P31939 & ATIC & Bifunctional purine biosynthesis protein & 2.98 & 0.0053 \\
\hline 060361 & NME2P1 & Putative nucleoside diphosphate kinase & 2.61 & 0.0077 \\
\hline P14625 & HSP9OB1 & Endoplasmin & 2.36 & 0.0325 \\
\hline P07910 & HNRNPC & Heterogeneous nuclear ribonucleoproteins C1/C2 & 2.32 & 0.0016 \\
\hline P55786 & NPEPPS & Puromycin-sensitive aminopeptidase & 2.24 & 0.0149 \\
\hline P61978 & HNRNPK & Heterogeneous nuclear ribonucleoprotein $\mathrm{K}$ & 2.06 & 0.0092 \\
\hline P14649 & MYL6B & Myosin light chain $6 \mathrm{~B}$ & 2.03 & 0.0148 \\
\hline P13639 & EEF2 & Elongation factor & 2.02 & 0.0002 \\
\hline P12004 & PCNA & Proliferating cell nuclear antigen & 1.99 & 0.0289 \\
\hline P00558 & PGK1 & Phosphoglycerate kinase & 1.94 & 0.0011 \\
\hline P49327 & FASN & Fatty acid synthase & 1.74 & 0.0039 \\
\hline P60709 & $A C T B$ & Actin & 1.68 & 0.0006 \\
\hline P07355 & ANXA2 & Annexin A2 & 1.67 & 0.0060 \\
\hline P23527 & HIST1H2BO & Histone H2B type 1-O & 1.63 & 0.0470 \\
\hline P29692 & EEF1D & Elongation factor 1-delta & 1.61 & 0.0162 \\
\hline P04908 & HIST1H2AB & Histone $\mathrm{H} 2 \mathrm{~A}$ type 1-B/E & 1.52 & 0.0208 \\
\hline P14618 & PKM & Pyruvate kinase & 1.43 & 0.0260 \\
\hline P29401 & $T K T$ & Transketolase & 1.32 & 0.0096 \\
\hline P06576 & ATP5B & ATP synthase subunit beta & 1.31 & 0.0025 \\
\hline
\end{tabular}


chromatin were observed to be the most enriched biological process, molecular function and cellular compartment respectively among the upregulated proteins (Figure 2).

\section{Protein-Protein Interaction and Pathway Analysis of Differ- entially Expressed Proteins}

In order to uncover underlying signaling pathways and associated proteins affected by flavonoid treated hormone positive breast cancer cells during their hormetic response, we carried out STRING, Reactome and KEGG pathway analysis with differentially expressed proteins. STRING results indicated three sig- nificant clusters related to carbon metabolism, biosynthesis of amino acids and spliceosome (Figures 3 and 4). In addition, Reactome analysis reported two more significantly altered pathways: mitochondrial protein import and eukaryotic translation elongation. The analysis revealed that the protein expressions (including proteins; G6PD: Glucose-6-phosphate 1-dehydrogenase, PGK1: Phosphoglycerate kinase, TKT: Transketolase, PKM: Pyruvate kinase) involved in carbon metabolism, amino acid synthesis (same proteins except G6PD) and eukaryotic translation elongation (EEF1D: Elongation factor 1-delta and EEF2: Elongation factor) were downregulated (except SHMT2: Serine
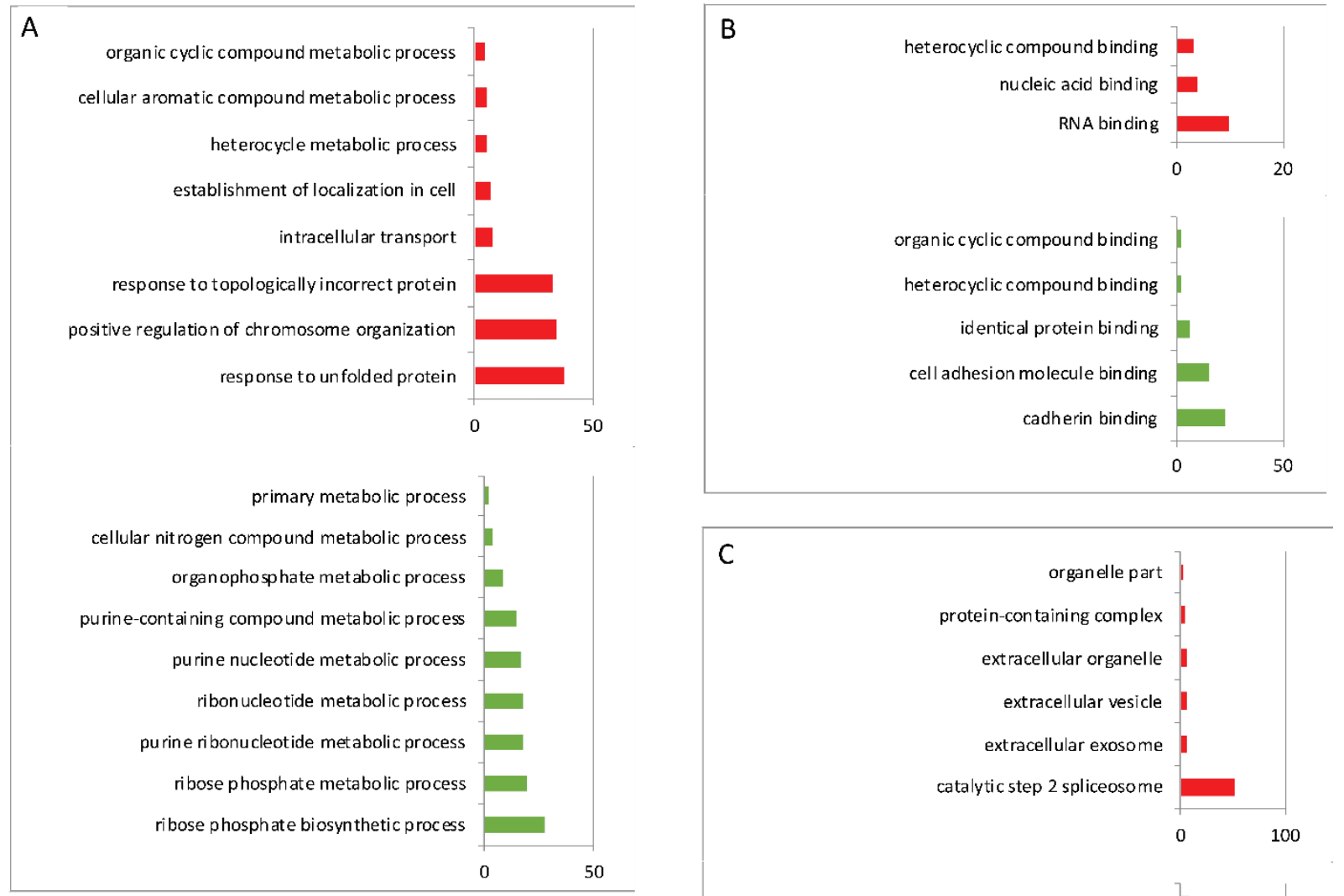

\section{C}

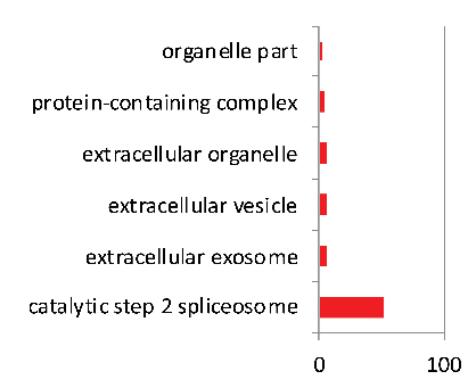

Fold enrichment upregulated

Fold enrichment downregulated

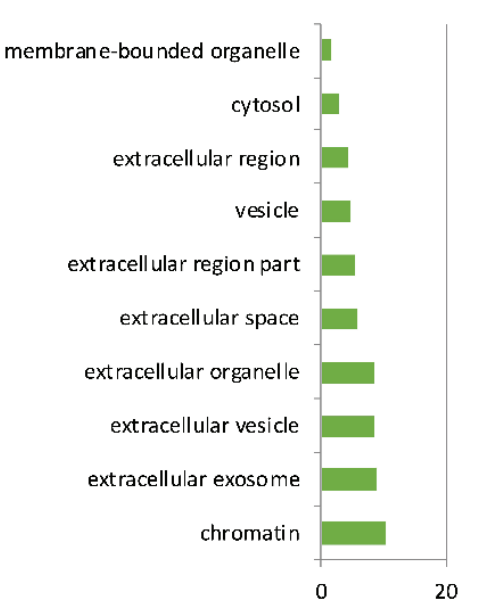

Figure 2. Gene Ontology (GO) enrichment analysis of significantly altered proteins. Biological process (A), molecular function (B) and cellular component $(A)$ are represented. Displaying only results for Bonferroni-corrected for $\mathrm{p}<0.05$. 


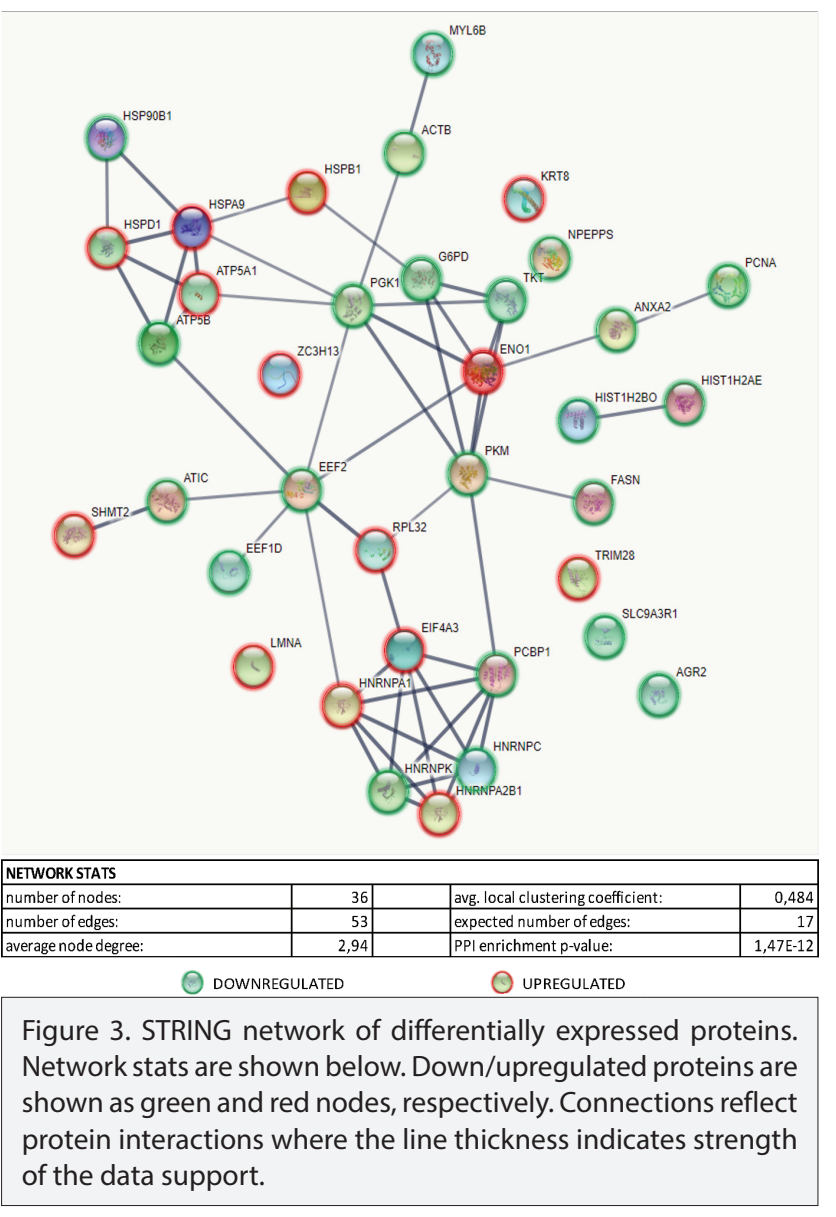

hydroxymethyl transferase, ENO1: Alpha enolase and RPL32: 605 ribosomal protein L32) in cells exposed to the low-dose FM. On the other hand, the proteins involved in the mitochondrial protein import pathway (HSPD1: $60 \mathrm{kDa}$ heat shock protein, HSPA9: Stress-70 protein, ATP5A1: ATP synthase subunit alpha) were observed to be upregulated (except ATP5B: ATP synthase subunit beta). Splicing mechanism was also observed to be altered, but not towards a specific direction.

\section{DISCUSSION}

Secondary metabolites originating from dietary flavonoids are well recognized for their wide range of pharmacological activities and even for their anticancer potential. Studies have shown that there is a positive correlation between a diet rich in flavonoids and a decrease in the risk of colon, prostate and breast cancer (19). In the light of these findings, it has raised the question whether flavonoids can be effective in the prevention and treatment of cancer by interacting with different genes and proteins. Therefore, the investigation of their effect on biological pathways along with their significant protein interactions is of utmost important.

Dietary flavonoids are also known to be among hormetic factors. Hormesis can simply be described as an observed biphasic dose response, which is stimulation at low doses and inhibition

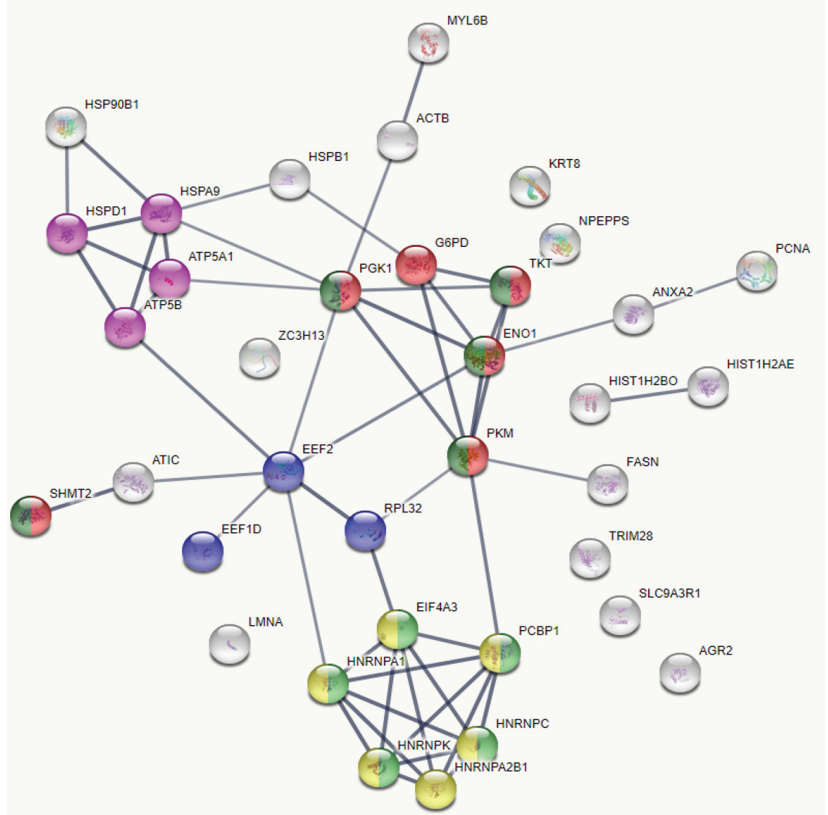

\begin{tabular}{|c|c|c|c|c|}
\hline \multicolumn{5}{|l|}{ KEGG } \\
\hline Pathway & Description & & Count in gene set & False discovery rate \\
\hline hsa01200 & Carbon metabolism & 둔 & 6 of 116 & $7,30 \mathrm{E}-06$ \\
\hline hsa01230 & Biosynthesis of amino acids & 를 & 5 of 72 & $1,19 \mathrm{E}-05$ \\
\hline hsa03040 & Spliceosome & : & 5 of 130 & 0,00013 \\
\hline \multicolumn{5}{|l|}{ REACTOME } \\
\hline Pathway & Description & & Count in gene set & False discovery rate \\
\hline HSA-72163 & mRNA Splicing - Major Pathway & $\theta$ & 6 of 178 & 0,00029 \\
\hline HSA-1268020 & Mitochondrial protein import & 른 & 4 of 64 & 0,00053 \\
\hline HSA-156842 & Eukaryotic Translation Elongation & e & 3 of 90 & 0,0122 \\
\hline
\end{tabular}

Figure 4. Significantly enriched pathways associated with proteins among the STRING network are shown. Top 3 pathways for KEGG and Reactome analysis are shown.

at high doses. When a cell receives a low dose of toxin, it tries to compensate with the disruption of cell homeostasis. In this process the cell activates its pathways for survival and promotes proliferation. In summary, hormesis is a stress response. Environmental factors such as heat and low-dose chemicals cause this effect.

In our study, a hormetic effect was observed as a result of the application of our patent-stage standardized flavonoid mixture, consisting of the flavonoids in propolis, on the MCF-7 cell line at a dose range of $16-117 \mu \mathrm{g} / \mathrm{mL}$. Due to the $16 \mu \mathrm{g} / \mathrm{mL}$ dose application, an increase of up to $35 \%$ in viability was observed in the cells, and a cytotoxic effect was observed in correlation with the increasing dose. The proteome analysis we performed to elucidate the mechanism of the hormetic effect observed at this dose indicated significant changes in carbon metabolism, amino acid biosynthesis, splicing mechanism, mitochondrial protein import and translation elongation pathways.

Today, the perception that cancer is a metabolic disease is getting stronger (20). Tumor cells are known to modulate their metabolism for growth, survival and proliferation (21). Since the times of Otto Warburg, the central carbon metabolism is known to have an impact in the progression of different types of cancers, in- 
cluding breast carcinomas $(22,23)$. High glucose uptake and lactate production under aerobic conditions in the tumor cells are supported by glycolysis and the pentose phosphate pathways (PPP). Regarding these dysregulated pathways, flavonoids are known to have anti-Warburg effects on cancer cells (24). Thus, it was not a surprise that we have identified altered expressions of proteins related to glycolysis and PPP. We observed expressions of the enzymes responsible for substrate level phosphorylation were downregulated (PGK1 FC=1.9, PKM FC=1.4) as well as PPP enzymes G6PD and TKT at the hormetic condition compared to the FM free cultured MCF-7 cells. In contrast, ENO1 and SHMT2 protein expressions were found to be upregulated.

Up to date data related to ENO1 expression levels in breast cancer cells/tissues were associated with the PI3K/Akt signaling pathway. Downregulation of ENO1 was shown to decrease the proliferation in breast cancer cells via the PI3K/Akt signaling pathway which is crucial for a series of cellular activities such as glycolysis (25). Carbon metabolism and amino acid metabolism are intimately connected to each other and share many proteins that have a role in both pathways. In this regard, SHMT2 is another key metabolic enzyme that converts serine to glycine.

Serine is necessary for synthesis of biomolecules such as proteins that are required for cell proliferation. By the activity of SHMT2, mitochondrial one-carbon metabolism produces $1 \mathrm{C}$ units from serine. After the oxidation of these $1 \mathrm{C}$ units, they are exported to the cytoplasm for the induction of one-carbon metabolism. Thus, one carbon metabolism serves as a set of reactions that supply methyl groups (one-carbon moiety) for de novo nucleotide biosynthesis and DNA methylation (26). It has been reported that SHMT2 was overexpressed in breast cancer as in many cancers. In addition, this situation was associated with tumorigenesis and progression $(27,28)$. Under the stress of low-dose FM, MCF-7 cells may reprogram their carbon and amino acid metabolism. Our findings correlate in the same direction with previous studies in which ENO1 and SMHT2 upregulation may be one of the responsible players that cause the hormetic effect of the FM.

Messenger RNA processing is a principal step for expression of eukaryotic genes that helps cells maintain various biological processes, such as proliferation, survival, and differentiation (2931). Many diseases, such as cancer development and progression, have been linked to anomalies in mRNA splicing (32). In breast cancer, unusual alternative splicing has been shown to be one of the risk factors. Furthermore, flavonoids such as apigenin and luteolin were shown to bind spliceosome components and therefore have an effect on splicing molecular mechanisms (33).

According to our analysis, expression levels of eukaryotic translation initiation factor 4A3 (EIF4A3) together with the heterogeneous nuclear ribonucleoproteins (hnRNPs) altered where the FM showed its hormetic effect. Deregulation of these alternative splicing elements have already been established as factors for breast cancer development $(34,35)$. Although the exact molecular mechanism is not known, spliceosome (HSA-03040) and the mRNA splicing pathway (HSA-72163) have a role where lowdose FM shows its hormetic effect on hormone positive breast cancer cells.

Many mitochondrial proteins encoded by nuclear genes are synthesized in the cytosol. These proteins are transported to the mitochondria in order to go through a delivery and sorting process by the mitochondrial import mechanism. Heat shock proteins (HSPs) acting as molecular protein chaperones have important roles in mitochondrial protein import. Specifically, after the cells have experienced different kinds of cellular stress, HSPs assist correct protein folding and sustain protein stability. They are also related with cancer development, progression, metastasis and drug resistance (36). Although flavonoids are known as inhibitors of HSPs expression (37), our data indicates that they can also induce expression of some of the proteins in this family at low doses because of their biphasic nature. A noteworthy hit in this pathway, mitochondrial stress protein 70 (HSPA9, mtHsp70, or mortalin), is a member of the heat shock protein 70 family. HSPA9 is known to prevent apoptosis via interacting with $\mathrm{p} 53$ and inactivating the tumor suppressor feature of p53 (38). In addition, it has been determined that increased expression of HSPA9 contributes to tumor formation $(39,40)$. Studies have shown that HSPA9 is a certain factor in breast cancer occurrence and development (41).

Moreover, HSPA9 plays an important role in the synthesis of iron-sulfur clusters (ISC) in mitochondria. Its function is to interact with and stabilize ISC cluster-assembly proteins which are used for various functions such as substrate binding and activation, electron transport, radical generation, regulation of gene expression, and DNA repair. Therefore, it can be speculated that the initial stress state caused by the low dose FM leads to a significant upregulation (7.8-fold) of this protein which may have contributed to MCF7 cell proliferation.

Mitochondrial protein import is fueled by ATP hydrolysis thus it is not surprising ATP synthase complex is affected from the FM treatment.

An upregulation of ATP synthase was detected in breast tumors, where also a correlation was observed between the a subunits (ATP5A1) and high stage, poorly differentiated and larger tumors $(42,43)$. However, according to Isidoro et al., there is no significant change in the expression levels of $\beta$ subunits (ATP5B) in breast cancer cells compared to normal breast cells (44). Nonetheless, decreased catalytic $\beta$ subunit expression has been associated with cancer development (45-47). Although we cannot suggest a certain direction of the expression (ATP5A1 1.3-fold up, ATP5B 1.3-fold down), it is possible to say that impairment of ATP synthesis takes part in the molecular mechanism of hormesis together with mitochondrial transport of proteins.

Translation is a complicated process that is disrupted through different mechanisms in cancer. (48). EEF2 and EEF1D, which were identified in our MS analysis, are such factors that play essential roles in the polypeptide chain elongation step. Ex- 
pression of elongation factors were shown to be significantly different within the subtypes of breast cancers, suggesting they have a potential of being therapeutic targets and prognostic biomarkers (49).

Increased EEF2 levels have been linked to poor outcome of HR (+) breast cancer (50). Furthermore, flavonoid structures can specifically bind to these elongation factors and can inhibit proliferation of breast cancer cells (51). We observed downregulation of EEF2 and EEF1D compared to untreated cells which favors antiproliferative effects of FM; however, it can be hypothesized that MCF-7 cells tend to overcome this via increasing ribosomal proteins such as RPL32 expression. RPL32 overexpression in breast cancer cells and tissues have been associated with cell migration and invasion (52). Although there has been no evidence of RPL32 protein and flavonoids interaction to our knowledge, we may suggest low-dose FM alters the translation elongation pathway through ribosomal protein expression levels in order to support the proliferation response of hormone positive breast cancer cells.

\section{CONCLUSION}

In our study we investigated the underlying biological pathways of the hormetic effect which was observed in FM treated MCF-7 cells. We have shown that multiple mechanisms are involved in the proliferation outcome of low-dose FM exposure. We suggest that the modulation of biological pathways, such as carbon metabolism, amino acid biosynthesis, splicing mechanism, mitochondrial protein import and translation elongation pathways are essential for the response of hormone positive breast cancer cells to flavonoids. Further studies on the hormetic effect of flavonoids on breast cancer in association with dietary intake values extrapolated from in vivo conditions are required to validate these preliminary findings. Elucidating the mechanisms that lie beneath the hormetic effect of flavonoids on HR (+) breast cancers, particularly the ones triggering the intrinsic adaptive response that leads to proliferation, may highlight some potential targets for better preventive and prophylactic interventions.

Acknowledgements: We thank Hülya Yılmaz Aydoğan and Oğuz Öztürk for useful discussions and invaluable opinions during the preparation of the manuscript. We also thank Emel Akgün for her support in wet-lab experiments.

Peer Review: Externally peer-reviewed.

Author Contributions: Conception/Design of Study- M.B.T.; Data Acquisition- A.B.C.; Data Analysis/Interpretation- M.B.T.; Drafting Manuscript- M.B.T.; Critical Revision of ManuscriptM.B.T.; Final Approval and Accountability- M.B.T., A.B.C.

Conflict of Interest: Authors declared no conflict of interest.

Financial Disclosure: This study was funded by the Scientific Projects Coordination Unit, Istanbul University (Project No. 22859).

\section{REFERENCES}

1. Sung H, Ferlay J, Siegel RL, Laversanne M, Soerjomataram I, Jemal A, et al. Global cancer statistics 2020: GLOBOCAN estimates of incidence and mortality worldwide for 36 cancers in 185 countries. CA Cancer J Clin 2021; 71: 209-49.

2. Rodríguez-García C, Sánchez-Quesada C, J Gaforio J. Dietary Flavonoids as Cancer Chemopreventive Agents: An Updated Review of Human Studies. Antioxidants 2019; 8(5): 137.

3. Toreti VC, Sato HH, Pastore GM, Park YK, Recent progress of propolis for its biological and chemical compositions and its botanical origin, Evid Based Complement Alternat Med 2013; 697390.

4. Banskota AH, Tezuka Y, Kadota S. Recent progress in pharmacological research of propolis. Phytother Res 2001; 15(7): 561-71.

5. Peeters PH, Keinan-Boker L, van der Schouw YT, Grobbee DE. Phytoestrogens and breast cancer risk: Review of the epidemiological evidence. Breast Cancer Res Treat 2003; 77: 171-83.

6. Lim DW, Giannakeas V, Narod SA. Survival Differences in Chinese Versus White Women With Breast Cancer in the United States: A SEER-Based Analysis. JCO Glob Oncol 2020; 6: 1582-92.

7. Rupasinghe HP, Ronalds CM, Rathgeber B, Robinson RA. Absorption and tissue distribution of dietary quercetin and quercetin glycosides of apple skin in broiler chickens. J Sci Food Agric 2010; 90(7): 1172-8.

8. Gonzales GB, Smagghe G, Grootaert C, Zotti M, Raes K, Van Camp J. Flavonoid interactions during digestion, absorption, distribution and metabolism: a sequential structure-activity/property relationship-based approach in the study of bioavailability and bioactivity. Drug Metab Rev 2015; 47(2): 175-90.

9. Seyhan MF, Yılmaz E, Timirci-Kahraman Ö, Saygılı N, Kısakesen Hi, Gazioğlu S, et al. Different propolis samples, phenolic content, and breast cancer cell lines: Variable cytotoxicity ranging from ineffective to potent. IUBMB Life 2019; 71(5): 619-31.

10. Bonofiglio D, Giordano C, De Amicis F, Lanzino M, Andò S. Natural Products as Promising Antitumoral Agents in Breast Cancer: Mechanisms of Action and Molecular Targets. Mini Rev Med Chem 2016; 16(8): 596-604.

11. Pal S, Konkimalla VB. Hormetic Potential of Sulforaphane (SFN) in Switching Cells' Fate Towards Survival or Death. Mini Rev Med Chem 2016; 16(12): 980-95.

12. Son TG, Camandola S, Mattson MP. Hormetic dietary phytochemicals. Neuromolecular Med 2008; 10(4): 236-46.

13. Jodynis-Liebert J, Kujawska M. Biphasic Dose-Response Induced by Phytochemicals: Experimental Evidence. Journal of Clinical Medicine 2020; 9(3): 718.

14. Narter F, Diren A, Kafkasli A, Eronat AP, Seyhan MF, Yilmaz-Aydogan $\mathrm{H}$, et al. Anatolian Propolis Prevents Oxalate Kidney Stones: Dramatic Reduction of Crystal Deposition in Ethylene-Glycol-Induced Rat Model. Rec Nat Prod 2018; 12(5): 445-59.

15. Huang KT, Chen YH, Walker AM. Inaccuracies in MTS assays: major distorting effects of medium, serum albumin, and fatty acids. Biotechniques 2004 Sep; 37(3): 406, 408, 410-2.

16. Wiśniewski JR, Zougman A, Nagaraj N, Mann M. Universal sample preparation method for proteome analysis. Nat Methods 2009; 6(5): 359-62.

17. Akgun E, Tuzuner MB, Sahin B, Kilercik M, Kulah C, Cakiroglu HN, et al. Proteins associated with neutrophil degranulation are upregulated in nasopharyngeal swabs from SARS-CoV-2 patients. PLoS One 2020; 15(10): e0240012

18. Carbon S, Ireland A, Mungall CJ, Shu S, Marshall B, Lewis S, AmiGO Hub; Web Presence Working Group. AmiGO: online access to ontology and annotation data. Bioinformatics 2009; 25(2): 288-9. 
19. Batra P, Sharma AK. Anti-cancer potential of flavonoids: recent trends and future perspectives. 3 Biotech 2013; 3(6): 439-59.

20. Coller HA. Is cancer a metabolic disease? Am J Pathol 2014; 184(1): 4-17.

21. Pavlova NN, Thompson CB. The emerging hallmarks of cancer metabolism. Cell Metab 2016; 23(1): 27-47.

22. Liberti MV, Locasale JW. The Warburg effect: how does it benefit cancer cells? Trends Biochem Sci 2016; 41(3): 211-8.

23. Richardson AD, Yang C, Osterman A, Smith JW. Central carbon metabolism in the progression of mammary carcinoma. Breast Cancer Res Treat 2008; 110(2): 297-307.

24. Samec M, Liskova A, Koklesova L, Samuel SM, Zhai K, Buhrmann C, et al. Flavonoids against the Warburg phenotype-concepts of predictive, preventive and personalised medicine to cut the Gordian knot of cancer cell metabolism. EPMA J 2020; 11(3): 377-98.

25. Zang HY, Gong LG, Li SY, Hao JG. Inhibition of a-enolase affects the biological activity of breast cancer cells by attenuating PI3K/Akt signaling pathway. Eur Rev Med Pharmacol Sci 2020; 24(1): 249-57.

26. Locasale JW. Serine, glycine and one-carbon units: cancer metabolism in full circle. Nat Rev Cancer 2013; 13: 572-83.

27. Possemato R, Marks KM, Shaul YD, Pacold ME, Kim D, Birsoy K, et al. Functional genomics reveal that the serine synthesis pathway is essential in breast cancer. Nature 2011; 476(7360): 346-50.

28. Liu C, Wang L, Liu X, Tan Y, Tao L, Xiao Y, et al. Cytoplasmic SHMT2 drives the progression and metastasis of colorectal cancer by inhibiting $\beta$-catenin degradation. Theranostics 2021; 11(6): 2966-86.

29. Maniatis T, Reed R. An extensive network of coupling among gene expression machines. Nature 2002; 416: 499-506.

30. Millevoi S, Vagner S. Molecular mechanisms of eukaryotic pre-mRNA 3' end processing regulation. Nucleic Acids Res 2009; 38: 275774.

31. Orphanides G, Reinberg D. A unified theory of gene expression. Cell 2002; 108: 439-51.

32. Scotti MM, Swanson MS. RNA mis-splicing in disease. Nat Rev Genet 2016; 17(1): 19-32.

33. Kurata M, Fujiwara N, Fujita Kl, Yamanaka Y, Seno S, Kobayashi H, et al. Food-Derived Compounds Apigenin and Luteolin Modulate mRNA Splicing of Introns with Weak Splice Sites. iScience 2019; 22: 336-52.

34. Geuens T, Bouhy D, Timmerman V. The hnRNP family: insights into their role in health and disease. Hum Genet 2016; 135(8): 851-67.

35. Silipo M, Gautrey H, Tyson-Capper A. Deregulation of splicing factors and breast cancer development. J Mol Cell Biol 2015; 7(5): 388401.

36. Calderwood SK, Gong J. Heat Shock Proteins Promote Cancer: It's a Protection Racket. Trends Biochem Sci 2016; 41(4): 311-23.

37. Hosokawa N, Hirayoshi K, Nakai A, Hosokawa Y, Marui N, Yoshida $M$, et al. Flavonoids inhibit the expression of heat shock proteins. Cell Struct Funct 1990; 15(6): 393-401.
38. Wadhwa R, Yaguchi T, Hasan MK, Mitsui Y, Reddel RR, Kaul SC. Hsp70 family member, mot-2/mthsp70/GRP75, binds to the cytoplasmic sequestration domain of the $\mathrm{p} 53$ protein. Exp Cell Res 2002; 274(2): 246-53.

39. Wadhwa R, Takano S, Kaur K, Deocaris CC, Pereira-Smith OM, Reddel RR, et al. Upregulation of mortalin/mthsp70/Grp75 contributes to human carcinogenesis. Int J Cancer 2006; 118(12): 2973-80.

40. Na Y, Kaul SC, Ryu J, Lee JS, Ahn HM, Kaul Z et al. Stress chaperone mortalin contributes to epithelial-mesenchymal transition and cancer metastasis. Cancer Res 2016; 76(9): 2754-65.

41. Zhang R, Meng Z, Wu X, Zhang M, Zhang S, Jin T. Mortalin promotes breast cancer malignancy. Exp Mol Pathol 2021;1 18:104593.

42. Huang TC, Chang HY, Hsu CH, Kuo WH, Chang KJ, Juan HF. Targeting therapy for breast carcinoma by ATP synthase inhibitor aurovertin B. J Proteome Res 2008; 7: 1433-44.

43. Pan J, Sun LC, Tao YF, Zhou Z, Du XL, Peng L, et al. ATP synthase ecto-alpha subunit: a novel therapeutic target for breast cancer. J Transl Med 2011; 9: 211.

44. Isidoro A, Casado E, Redondo A, Acebo P, Espinosa E, Alonso AM, et al. Breast carcinomas fulfill the Warburg hypothesis and provide metabolic markers of cancer prognosis. Carcinogenesis 2005; 26: 2095-104.

45. Willers IM, Cuezva JM. Post-transcriptional regulation of the mitochondrial $\mathrm{H}(+)$-ATP synthase: a key regulator of the metabolic phenotype in cancer. Biochim Biophys Acta 2011; 1807: 543-51.

46. Cuezva JM, Krajewska M, de Heredia ML, Krajewski S, Santamaría $\mathrm{G}, \mathrm{Kim} \mathrm{H}$, et al. The bioenergetic signature of cancer: a marker of tumor progression. Cancer Res 2002; 62: 6674-81.

47. Zheng SQ, Li YX, Zhang Y, Li X, Tang H. MiR-101 regulates HSV-1 replication by targeting ATP5B. Antiviral Res 2011; 89: 219-26.

48. Bilanges B, Stokoe D. Mechanism of translational deregulation in human tumors and therapeutic intervention strategies. Oncogene 2007; 26: 5973-90.

49. Hassan MK, Kumar D, Naik M, Dixit M. The expression profile and prognostic significance of eukaryotic translation elongation factors in different cancers. PLoS One 2018; 13(1): e0191377.

50. Meric-Bernstam F, Chen H, Akcakanat A, Do KA, Lluch A, Hennessy BT, Hortobagyi GN, Mills GB, et al. Aberrations in translational regulation are associated with poor prognosis in hormone receptor-positive breast cancer. Breast Cancer Res 2012; 14: R138.

51. Yao N, Chen CY, Wu CY, Motonishi K, Kung HJ, Lam KS. Novel flavonoids with antiproliferative activities against breast cancer cells. J Med Chem 2011; 54(13): 4339-49.

52. Xu L, Wang L, Jiang C, Zhu Q, Chen R, Wang J, et al. Biological effect of ribosomal protein L32 on human breast cancer cell behavior. Mol Med Rep 2020; 22(3): 2478-86. 